\title{
Dieta del aguilucho común Geranoaetus polyosoma (Quoy \& Gaimard 1824) en la Región de Atacama, Chile
}

\author{
Diet of variable hawks Geranoaetus polyosoma (Quoy \& Gaimard 1824) in Atacama \\ Region, Chile
}

\author{
Pablo Valladares Faúndez ${ }^{1 *}$, Nicole Álvarez Henriquez ${ }^{2}$, Natalia Urrutia Osorio², Flavio Olivares \\ Zuleta $^{3}$ \& Sergio Alvarado Orellana ${ }^{4}$
}

${ }^{1}$ Departamento de Biología, Facultad de Ciencias, Universidad de Tarapacá. Avenida General Velásquez 1775, Arica, Chile. ${ }^{2}$ Universidad de La República, Rafael Sotomayor 420, Arica

${ }^{3}$ Tierra del Sol Investigación y Desarrollo Consultores. Añañuca 799, Quinta Valle, Vallenar, Chile. ${ }^{4}$ Laboratorio de Ecología de Vida Silvestre, Facultad de Ciencias Forestales y de la Conservación de la Naturaleza, Universidad de Chile. Av. Santa Rosa 11315, Santiago, Chile.

*pvalladares@uta.cl

\section{RESUMEN}

El aguilucho común (Geranoaetus polyosoma) es una especie de ave rapaz que se distribuye desde Arica a Tierra del Fuego en Chile. Se alimenta de pequeños vertebrados e invertebrados, particularmente insectos y arácnidos. No existe suficiente información biológica de esta especie en el norte de Chile, desconociéndose su reproducción, dieta, etc. En este trabajo evaluamos la dieta del aguilucho común, por medio del análisis de egagrópilas colectadas entre las localidades de Vallenar y Copiapó, Región de Atacama, la que corresponde a una zona híperárida. Esta información fue comparada con la literatura científica existente en el resto del país y países limítrofes. Dada las condiciones ambientales de extrema aridez, esperamos encontrar una baja diversidad de presas, situación característica de las rapaces de ecosistemas áridos. En el caso particular de las poblaciones de aguilucho en la Región de Atacama el ítem presa más frecuente fue reptiles (57,1\%) el que correspondió a lagartos pequeños del género Liolaemus spp (30,4\%), junto con L. bisignatus (17\%) y Callopiste maculatus $(5,3 \%)$. Los ítems presa secundarios correspondieron a roedores (19,8\%) y coleópteros del género Gyriosomus $(18,9 \%)$. No hubo correlación positiva entre la frecuencia de presas y su masa corporal $\left(\mathrm{r}^{2}=-0,267, \mathrm{p}>0,05\right)$ lo que indica que esta rapaz no selecciona a sus presas por tamaño. La biomasa de reptiles en la dieta $(55,3 \%)$ fue levemente mayor a mamíferos (42,7\%), pero quién más aportó fue Phyllotis darwini con un 27,6\%. Tanto el Índice de Simpson (IS=0,1833) como el Índice de Shannon $\left(\mathrm{H}^{\prime}=0,8463\right)$ indican que esta especie tiene una baja diversidad de presas, lo que está en concordancia con otras rapaces habitantes en ecosistemas áridos.

Palabras Clave: Geranoaetus polyosoma; dieta; ambientes áridos; Liolaemus; Chile; Desierto de Atacama.

\begin{abstract}
The Variable Hawk (Geranoaetus polyosoma) is a raptor species distributed from Arica to Tierra del Fuego in Chile. It consumes small vertebrates and invertebrates, particularly insects and arachnids. The biological information of this species is scarce for northern Chile, particularly about their reproduction, diet, etc. We evaluated the diet of the Variable Hawk, by means of pellets collected between Vallenar to Copiapó, in Atacama region, a hyper-arid area. This information was compared with the available scientific literature in Chile and neighboring countries. Because of the environmental conditions of extreme aridity, we expect to find a low diversity of prey, characteristic of the raptors of arid ecosystems. In the case of the Variable Hawk populations from Atacama region, the most consumed species were reptilians $(57,1 \%)$ corresponding to small lizards of the Liolaemus spp (30,4\%), L. bisignatus (17\%) and Callopiste maculatus $(5,3 \%)$. Other prey corresponded to rodents (19.8\%) and coleopterans of the genus Gyriosomus (18.9\%). There was no positive correlation between frequency of prey and their body mass $\left(r^{2}=-0.267, p>0.05\right)$ indicating that this raptor does not select their prey by body size. The biomass of reptiles in the diet $(55.3 \%)$ was slightly higher than mammals (42.7\%), but the largest individual contribution was given by Phyllotis darwini with $27.6 \%$. Both the Simpson $(\mathrm{SI}=0.1833)$ and the Shannon index $\left(\mathrm{H}^{`}=0.8463\right)$ indicate that this species consume a low diversity of prey, which is consistent with the others raptors that inhabit arid environments.
\end{abstract}

KeYwords: Geranoaetus polyosoma; diet; arid environment; Liolaemus; Chile; Atacama Desert. 


\section{INTRODUCCIÓN}

El aguilucho común (Geranoaetus polyosoma) es una especie de rapaz de la Familia Accipitridae (Thiollay 1994), que presenta una amplia distribución en gran parte de Sudamérica, encontrándose por el norte desde la cordillera central de Colombia hasta el Cabo de Hornos por el sur, y desde las costas de Chile hasta Argentina, (Brown \& Amadon 1968, Cabot 1991, Vaurie 1962, De Lucca 2011), llegando a las Islas Malvinas en el Atlántico (Cabot 1991) y a las Islas Juan Fernández por el Pacífico (Blake 1997). Su biología es poco conocida (Jiménez 1995) a pesar de ser una de las rapaces más estudiadas en Chile (Raimilla et al. 2012), donde la información disponible aún es escasa, particularmente en el norte de Chile (Cabot et al. 2010). Habita desde el nivel del mar hasta los 4000 metros de altitud en la Cordillera de Los Andes (Goodall et al. 1951), es extremadamente variable en su color, teniendo dimorfismo sexual y ontogenético (Olrog 1959, Vaurie 1962, Brown \& Amadon 1968). Es de mediano tamaño, con una envergadura de $1100 \mathrm{~mm}$, longitud del cuerpo entre 450 y $530 \mathrm{~mm}$ para los machos y 485 y $630 \mathrm{~mm}$ para hembras, pero machos adultos entre 690 y 1134 gr y hembras adultas entre 876 y 1417 gr (Jiménez 1995). En la zona central de Chile esta especie comienza su período de reproducción en agosto (Barros 1962), nidifica principalmente en quebradas, aunque en Atacama se encuentran muchos nidos activos e inactivos en torres de alta tensión (Jimenez 1995). En Ecuador y Perú se han descrito nidificaciones en cactus (Marchant 1960), lo que también se observa en el desierto de Atacama en Chile, pero ninguno activo.

En relación a su dieta, la información es parcialy fragmentada. Se ha documentado para la patagonia argentina (Monserrat et al. 2005, Travaini et al. 2012) y chilena (Figueroa et al. 2003), para la Provincia de Buenos Aires (Baladrón et al.
2006, 2009), zona centro-norte de Chile (Schlatter et al. 1980, Jiménez 1995) y la Isla Juan Fernández (Fuentes et al. 1993), observándose una preferencia por roedores, y secundariamente por aves, reptiles e invertebrados (Tabla 1). Sin embargo, no hay antecedentes de las características tróficas de esta especie en el norte de Chile. Por tal motivo en este trabajo nos proponemos analizar la dieta del aguilucho común en la Región de Atacama, particularmente en un lugar que corresponde a una zona hiperárida y que es considerado como Sitio Prioritario de Conservación "Desierto Florido" (Squeo et al. 2008).

\section{MATERIAL Y MÉTODO}

El estudio se realizó entre las ciudades de Vallenar a Copiapó, Región de Atacama, Chile. Las condiciones ecológicas del desierto de interior de la Región de Atacama corresponde a una zona hiperarida (Juliá et al. 2008) denominada Desierto Florido de los Llanos (Gajardo 1994), con un Clima Desértico Transicional (Novoa et al. 2008, Juliá et al. 2008) y geomorfológicamente denominada Pampa Transicional (Novoa et al. 2008). Desde el punto de vista del paisaje ecogeográfico, la zona de estudio contempla dos áreas denominadas Pampeana y Serrano, con las más bajas precipitaciones de la región $(<0,5 \mathrm{~mm}$ en condiciones normales).

Se recolectaron egagrópilas encontradas bajo nidos y perchas ubicados en las torres de alta tensión (Tabla 2). Cada egagrópila recolectada fue guardada en una bolsa de papel con un código numérico que incluye el número de torre analizada y el número de egagrópila de dicha torre. Las egagrópilas fueron trasladadas al Laboratorio de Zoología Integrativa de la Universidad de Tarapacá, Arica, Chile. Las egagrópilas fueron hidratadas individualmente por tres

TABla 1. Porcentaje (\%) de presas consumidas por Geranoaetus polyosoma en distintas partes de Chile y Argentina. †: Jiménez 1995, †: Schlatter et al. 1980, *: Figueroa et al. 2003, \#: Traviani et al. 2012, §: Monserrat et al. 2005, ¥: Baladrón et al. 2006.

TABLE 1. A percentage (\%) of prey consumed for Geranoaetus polyosoma in different parts of Chile and Argentina. †: Jiménez 1995, †: Schlatter et al. 1980, *: Figueroa et al. 2003, \#: Traviani et al. 2012, §: Monserrat et al. 2005, ¥: Baladrón et al. 2006.

\begin{tabular}{lccccccc}
\hline \multicolumn{5}{c}{ Chile } & \multicolumn{5}{c}{ Argentina } \\
\hline & Aucó $^{\dagger}$ & La Dehesa $^{\ddagger}$ & Aysén* $^{*}$ & MN Bosque Petrificado $^{\#}$ & Junín de Los Andes $^{\#}$ & Provincia de Neuquén $^{\S}$ & Buenos Aires $^{*}$ \\
\hline \hline Roedores & 45 & 89,3 & 82 & 66,44 & 47,5 & 82,4 & 99,5 \\
Reptiles & 20 & 0,3 & 0 & 5,56 & 25,19 & 7,1 & 0 \\
Aves & 6,6 & 3,3 & 4,7 & 4,86 & 1,67 & 2,1 & 0,5 \\
Insectos & 0 & 0 & 5,7 & 19,68 & 8,46 & 3,1 & 0 \\
Arácnidos & 0 & 0 & 0 & 1,62 & 0 & 0 & 0 \\
NI & 0 & 0 & 7,6 & 0 & 0 & 1,2 & 0 \\
\hline
\end{tabular}


horas para posteriormente separar los restos óseos como cráneos y mandíbulas. El resto del material (pelos, piel y otros restos biológicos) se filtró, secó y guardó en bolsas de papel con el mismo código numérico.

El análisis de los fragmentos óseos y dentales fue realizado mediante la lupa binocular Olympus XTL-2310. Para el reconocimiento de las especies presas, se utilizaron claves de molares en el caso de los roedores (Muñoz-Pedreros \& Gil 2009, Yáñez et al. 2009), y muestras de referencias de roedores y lagartos que habitan la Región de Atacama, los que se encuentra en la Colección Zoológica de Zonas Áridas y Andinas de la Universidad de Tarapacá (CZZA - UTA). Cada una de las presas fue registrada en una base de datos y se desarrolló un análisis de frecuencia y abundancia de presas en cada egagrópila, además de la biomasa aportada por cada ítem presa, tomándose como referencia a Cortés et al. (1992) para Liolaemus y Callopistes, Spotorno et al. (1998) para Oligoryzomys, Abrothrix y Abrocoma, y Spotorno et al. (2013) para Eligmodontia. Posteriormente se desarrolló una correlación lineal de Pearson entre la frecuencia de presas y la masa corporal por ítem presa, con la finalidad de evaluar preferencia de dieta por tamaño corporal de las presas. Finalmente se calcularon los índices de Simpson y Shannon para evaluar la riqueza de presas consumidas (Muños-Pedreros \& Rau 2004). Los análisis estadísticos se realizaron en el programa SPSS 10.0.

\section{RESULTADOS}

Se analizaron 198 egagrópilas de Aguilucho Común, resultando un total de 359 presas, las que fueron identificadas por medio de restos mandibulares, cráneos, exoesqueletos y piel. Solo los lagartos del Género Liolaemus de pequeño tamaño no fueron asignadas a un estatus específico, ya que no presentan caracteres diagnósticos a nivel de cráneo o mandíbula y fue imposible determinarlas a nivel de especie, por lo que son determinadas como Liolaemus spp. El grupo mayormente consumido fueron precisamente los lagartos, representando un $56 \%$. De estos, el mayor ítem presa corresponde especies de lagartos pequeños y que podrían corresponder a Liolaemus atacamanesis, L. platei y $L$. velosoi (Valladares 2011). Este ítem presa corresponde al $30,4 \%$, mientras que L. bisignatus, lagarto de mayor tamaño, correspondió al 17,1\% y Callopistes maculatus con un 5,3\% de la dieta del aguilucho común (Tabla 3). El segundo ítem en importancia fue el artrópodo del género Gyriosomus con un $18,9 \%$. En el caso de los roedores, Phyllotis darwini fue la especie más consumida, con un $13,9 \%$.

TABLA 2. Coordenadas de las torres de alta tensión donde se colectaron las egagrópilas de Geranoaetus polyosoma, entre las ciudades de Vallenar y Copiapó, Región de Atacama, Chile.

TABLE 2. Coordinates of the high tension towers where pellets of Geranoaetus polyosoma are collected, between the cities of Vallenar and Copiapo, Atacama Region, Chile.

\begin{tabular}{|c|c|c|}
\hline $\mathrm{N}^{\circ}$ TORRE & CoOrdenadas & $\mathrm{N}^{\circ}$ EGAGROPILAS \\
\hline 1 & $28^{\circ} 30^{\prime} 44.65^{\prime \prime} \mathrm{S} ; 70^{\circ} 52^{\prime} 49.61^{\prime \prime} \mathrm{O}$ & 3 \\
\hline 2 & $28^{\circ} 30^{\prime} 41.90^{\prime \prime} \mathrm{S} ; 70^{\circ} 52^{\prime} 37.52^{\prime \prime} \mathrm{O}$ & 1 \\
\hline 3 & $28^{\circ} 25^{\prime} 9.62 ” \mathrm{~S} ; 70^{\circ} 46^{\prime} 57.78^{\prime \prime O}$ & 3 \\
\hline 4 & $28^{\circ} 24^{\prime} 58.82 ” S ; 70^{\circ} 46 ’ 52.97 ” O$ & 2 \\
\hline 5 & $28^{\circ} 24^{\prime} 49.10^{\prime \prime S} ; 70^{\circ} 46^{\prime} 48.09 ” \mathrm{O}$ & 5 \\
\hline 6 & $28^{\circ} 24^{\prime} 38.26^{\prime \prime} \mathrm{S} ; 70^{\circ} 46^{\prime} 42.76^{\prime \prime} \mathrm{O}$ & 21 \\
\hline 7 & $28^{\circ} 24^{\prime} 28.19^{\prime \prime} \mathrm{S} ; 70^{\circ} 46^{\prime} 37.39^{\prime \prime O}$ & 36 \\
\hline 10 & $28^{\circ} 13^{\prime} 3.54 ” \mathrm{~S} ; 70^{\circ} 40^{\prime} 52.43 ” \mathrm{O}$ & 36 \\
\hline 11 & $28^{\circ} 10^{\prime} 50.48^{\prime \prime} \mathrm{S} ; 70^{\circ} 39^{\prime} 38.29{ }^{\prime} \mathrm{O}$ & 24 \\
\hline 12 & $28^{\circ}$ 7’17.26”S; 70³7’34.04”O & 28 \\
\hline 13 & $28^{\circ}$ 6’42.52”S; 70³7’13.88”O & 10 \\
\hline 14 & $28^{\circ} 5{ }^{\prime} 33.37^{\prime \prime} \mathrm{S} ; 70^{\circ} 36^{\prime} 34.11^{\prime \prime O}$ & 9 \\
\hline 15 & $28^{\circ} 3{ }^{\prime} 49.03 ” \mathrm{~S} ; 70^{\circ} 35^{\prime} 34.28^{\prime \prime} \mathrm{O}$ & 4 \\
\hline 16 & $28^{\circ} 3{ }^{\prime} 49.04^{\prime \prime S} ; 70^{\circ} 35^{\prime} 34.26^{\prime \prime} \mathrm{O}$ & 4 \\
\hline 17 & $28^{\circ} 3^{\prime} 25.08^{\prime \prime} \mathrm{S} ; 70^{\circ} 35^{\prime} 26.41^{\prime \prime} \mathrm{O}$ & 4 \\
\hline 18 & $28^{\circ} 6 ’ 21.50^{\prime \prime} \mathrm{S} ; 70^{\circ} 36^{\prime} 36.12^{\prime \prime} \mathrm{O}$ & 3 \\
\hline
\end{tabular}


Los aportes en biomasa fueron Phyllotis darwini con un $26,7 \%$ del total, Liolaemus bisignatus con un 21,9\%, Liolaemus spp con 16,6\% y Callopistes maculatus con $14,3 \%$. El grupo que más aporta a la biomasa total es Reptilia con un 55,3\%, seguida de Mammalia con un 42,7\%y Artrópoda solo un 2\% (Tabla 4). No hubo relación entre la masa corporal de las presas y su frecuencia en las egagrópilas $\left(r^{2}=-0,267, p=0,455\right)$. Finalmente, el Índice de Simpson $(\mathrm{IS}=0,1833)$ y el Índice de Shannon $\left(\mathrm{H}^{\prime}=0,8463\right)$ indican una baja diversidad de presas.

TABla 3. Abundancia y frecuencia de los ítemes presas consumidos por Geranoaetus polyosoma en el Desierto de Atacama, Región de Atacama. na = número de individuos consumidos. $\mathrm{nf}$ = número de egagrópilas en que se encontró dicha presa.

TABLE 3. Abundance and frequency of prey items consumed for Geranoaetus polyosoma in the Atacama Desert, Atacama Region. na = number of individuals consumed. $\mathrm{nf}=$ number of pellets in which the dam was found.

\begin{tabular}{lcccc}
\hline \multicolumn{1}{c}{ ITEM PRESA } & \multicolumn{2}{c}{ ABUNDANCIA } & \multicolumn{2}{c}{ FRECUENCIA } \\
\hline \multicolumn{1}{c}{ Rodentia } & $\mathrm{Na}$ & $\%$ & $\mathrm{Nf}$ & $\%$ \\
Abrocoma bennetti & 4 & 1,1 & 4 & 2,0 \\
Oligoryzomys longicaudatus & 1 & 0,3 & 1 & 0,5 \\
Phyllotis darwini & 50 & 13,9 & 50 & 25,5 \\
Abrothrix olivaceus & 3 & 0,8 & 3 & 1,5 \\
Eligmodontia dunaris & 4 & 1,1 & 4 & 2,0 \\
roedores no identificados $\quad$ Reptilia & 13 & 3,6 & 13 & 6,6 \\
Liolaemus spp. & & & & 54,8 \\
Liolaemus bisignatus & 109 & 30,4 & 106 & 31,1 \\
Callopistes maculatus & 61 & 17,1 & 61 & 9,7 \\
lagartos no identificados & 19 & 5,3 & 19 & 8,2 \\
vertebrado no identificados & 16 & 4,5 & 8 & 4,1 \\
Coleoptera Arthropoda & 8 & 2,2 & 66 & 33,7 \\
Scorpionida & & & 3 & 1,5 \\
\hline
\end{tabular}

Tabla 4. Biomasa aportada por cada ítems de presa en la dieta de Geranoaetus polyosoma. Promedios de masa corporal por especie recogidos de Mann (1978), Cofré y Marquet (1999), Muñoz-Pedreros y Yañez (2009) e Iriarte (2008) para los mamíferos; PincheiraDonoso y Núñez (2005), Cortés et al (1992) para reptiles y artrópodos datos propios.

TABLE 4. Biomass contributed by each of prey items in the diet of Geranoaetus polyosoma. Averages of body mass by species gathered from Mann (1978), Cofré and Marquet (1999), Muñoz-Pedreros and Yañez (2009) and Iriarte (2008) for mammals; Pincheira-Donoso and Nuñez (2005), Cortés et al (1992) for reptiles and arthropods own data.

\begin{tabular}{|c|c|c|c|c|}
\hline & $\mathrm{N}^{\mathrm{o}}$. DE ÍTEMS & masa/sp (gr) & TOtal Biomasa (gr) & $\%$ BIOMASA \\
\hline \multicolumn{5}{|l|}{ Mammalia } \\
\hline Abrocoma bennetti & 4 & 250,5 & 1002 & 9,6 \\
\hline Oligoryzomys longicaudatus & 1 & 27,8 & 27,8 & 0,3 \\
\hline Phyllotis darwini & 50 & 57,5 & 2875 & 27,6 \\
\hline Abrothrix olivaceus & 3 & 28 & 84 & 0,8 \\
\hline Eligmodontia dunaris & 4 & 11,9 & 47,6 & 0,5 \\
\hline roedores no identificados & 13 & 31,3 & 406,9 & 3,9 \\
\hline \multicolumn{5}{|l|}{ Reptilia } \\
\hline Liolaemus sp. & 109 & 15,86 & 1728,74 & 16,6 \\
\hline Liolaemus bisignatus & 61 & 37,4 & 2281,4 & 21,9 \\
\hline Callopistes maculatus & 19 & 78,2 & 1485,8 & 14,3 \\
\hline lagartos no identificados & 16 & 15,86 & 253,76 & 2,4 \\
\hline vertebrado no identificados & 8 & 0 & 0 & 0,0 \\
\hline \multicolumn{5}{|l|}{ Arthropoda } \\
\hline Coleoptera & 68 & 3 & 204 & 2,0 \\
\hline \multirow[t]{2}{*}{ Scorpionida } & 3 & 3 & 9 & 0,1 \\
\hline & 359 & & 10406 & \\
\hline
\end{tabular}




\section{DISCUSIÓN}

Nuestro trabajo corresponde al primer registro de la dieta del aguilucho común en el norte de Chile. La dieta de esta ave rapaz en el desierto de Atacama está compuesta principalmente por lagartos del género Liolaemus, seguida de Phyllotis darwini y artrópodos. Estos datos se contraponen a los encontrados en la literatura, ya que en los otros estudios la preferencia de dieta es por micromamíferos, valores que van desde un 45\% en Aucó, Chile (Jiménez 1995), hasta un 99,5\% en la Provincia de Buenos Aires, Argentina (Baladrón et al. 2006). Sin embargo, la mayor biomasa aportada en la dieta del aguilucho común en Atacama es de P. darwini.

Observando los ítem presa en una gradiente latitudinal, es posible encontrar especies cercanas a los valles de Vallenar y Copiapó, que no se encuentran en el desierto intermedio. Por ejemplo, en las torres de alta tensión cercanas al valle de Vallenar se observan especies presa como Abrocoma bennetti y Oligoryzomys longicaudatus, y en el valle de Copiapó aparece $O$. longicaudatus, pero no Abrocoma, a pesar de ser descrito para ese valle (Valladares \& Campos 2012). Sin embargo, las especies presa más frecuentemente encontradas en la zona desértica entre ambos valles, son $P$. darwini y Eligmodontia dunaris, ambas especies previamente descritas para esa zona (Valladares 2012, Spotorno et al. 2013).

En el caso de los lagartos, los Liolaemus de tamaño pequeños, como $L$. velosoi, $L$. atacamensis y $L$. platei carecen de caracteres diagnósticos a nivel mandibular, lo que impide distinguirlos a nivel de especie. Sin embargo, podemos afirmar que $L$. velosoi es una especie que está restringida al valle de Copiapó y habita zonas de matorrales, mientras que $L$. platei es más frecuente en roquedales, y L. atacamensis habita zonas de dunas (Valladares 2011). L. bisignatus es distinguible particularmente por su tamaño, mucho mayor que los otros Liolaemus, y Callopistes maculatus es distinguible por la forma característica de sus dientes cónicos.

Los Índices de Simpson y Shannon dieron valores bajos, lo que indica una baja diversidad de presas del Geranoaetus polyosoma en la Región de Atacama, comparada con poblaciones de la Patagonia chilena y argentina, donde el número de especies se incrementa hasta más del doble. Esta situación podría indicar una posible especialización del depredador en un ecosistema árido, lo que implicaría además una adaptación estratégica particular del depredador para aumentar la eficiencia de caza, es decir, una especialización comportamental (Devictor et al. 2010). Sin embargo, considerando variables indirectas tales como el gran rango de distribución de la especie y su nicho trófico fundamental (Hutchinson 1957), visualizado en la amplia gama de presas que tiene en su rango de distribución (Futuyma \& Moreno
1988, Calenge \& Basille 2008, Kassen 2002) nos podrían estar indicando una especie ecológicamente generalista, y estos resultados estén indicando una respuesta funcional a la disponibilidad de presas (Jaksic et al. 1992, Farias \& Jaksic 2007). Esta segunda hipótesis también se refuerza considerando la capacidad de adaptabilidad del aguilucho común a ambientes fuertemente fluctuantes (Levins 1968), como por ejemplo el Desierto de Atacama. Cabe mencionar en relación a esta segunda hipótesis, que al no existir relación entre el tamaño de las presas y su frecuencia en las egagrópilas, el aguilucho no estaría depredando selectivamente por el tamaño de las presas, sino más bien sería una situación circunstancial.

Llama particularmente la atención que el aguilucho común, teniendo tan amplio rango de distribución (Cabot 1991, Bellatti 2000, Pavez 2004, Pacheco 2004) haya sido poco estudiado en su biología trófica. Hemos podido constatar sólo seis estudios cuantitativos de dieta de esta especie, restringidos a Argentina y Chile. Los más antiguos sólo corresponden a observaciones ocasionales (Jiménez 1995). Las diferencias en las proporciones de mamíferos y lagartos consumidos puede tener relación con la coincidencia en la actividad diaria del aguilucho, ya que la mayoría de las especies de roedores encontradas presentan actividades nocturnas más que crepusculares (Iriarte 2008) y hemos observado reiteradamente a aguiluchos adultos dar caza a lagartos cerca del mediodía.

La importancia de este estudio radica en que esta población de aguiluchos habita en el Sitio Prioritario de Conservación "Desierto Florido" (Squeo et al. 2008), esto debido a que cuando ocurre el fenómeno de El Niño (ENSO), aumentan las precipitaciones y esta zona florece de manera muy significativa, ocurriendo cambios importantes en las poblaciones que ahí habitan. Por lo tanto, este trabajo corresponde a un primer diagnóstico de la biología dietaria del aguilucho común en la Región de Atacama, y que será evaluado al momento que ocurra un fenómeno ENSO, lo que nos permitirá tener una idea más clara de las consecuencias de este fenómeno en las comunidades biológicas del desierto de Atacama. Finalmente, consideramos que es probable que la utilización de las torres de alta tensión en la nidificación de esta especie sea un factor relevante para considerar su estado de conservación local, dado que dichas plataformas estarían dando una adecuada arquitectura para su nidificación y parece ser importante para su supervivencia.

\section{AGRADECIMIENTOS}

Este estudio fue financiado por el proyecto Universidad de Tarapacá / UTA Myor de Investigación Científica y tecnológica, Proyecto Nº4710 - 2015. 


\section{BIBLIOGRAFÍA}

Baladron, A.V., BO, M.S. \& Malizia A.I. 2006. Winter diet and time-activity budgets of the Red-Backed Hawk (Buteo polyosoma) in the coastal grasslands of Buenos Aires province, Argentina. Journal Raptor Research 40(1):65-70.

Baladron, A.V., BO, M.S. \& Malizia, A.I. 2009. Predation upon the subterranean rodent Ctenomys talarum (tuco-tucos) by Buteo polyosoma (Red-backed Hawks) in coastal grasslands of Argentina. Studies on Neotropical Fauna and Environment 44:61-65.

Barros, R. 1962. Apuntes acerca del Peuco y Aguilucho común. Revista Universitaria (Chile) 47:219-227.

Bellati, J. 2000. Comportamiento y abundancia relativa de rapaces de la Patagonia extraandina Argentina.Ornitología Neotropical 11:207-222.

Brown, L. \& Amadon, D. 1968. Eagles, hawks and falcons of the world. London: Country Life Books.

САвот, J. 1991. Distribution and habitat selection of Buteo polyosoma and $B$. poecilochrous in Bolivia and neighbouring countries. Bulletin British. Ornithologist. Club. 114:199-209.

Cabot, J., De Vries, T., \& Alvarado, S. 2010. Distribución espacial de rapaces en el Desierto de Atacama, Chile, con notas sobre el Busardo de Gurney Buteo poecilochrous. En: Aves Rapaces y Conservación. Una Perspectiva Iberoamericana (Eds. Hernandez, V.J., Muñiz, R., Cabot, J. \& de Vries, T.), pp. 153-162. Tundra Ediciones, Valencia, España.

Calenge, C. \& Basille, M. 2008. A general framework for the statistical exploration of the ecological niche. Journal of Theoretical Biology 252:674-685.

Cortés, A., Baez, C., Rosemann, M. \& Pino, C. 2008. Dependencia térmica del teiido Callopiste palluma: Una comparación con los iguánidos Liolaemus nigromaculatus y L. nitidus. Revista Chilena de Historia Natural 65:443-451.

De Lucca, E.R. 2011. Observaciones del aguilucho común (Buteo polyosoma) en el centro y sur de la Argentina. Nótulas Faunísticas 77:1-15.

Devictor, V., Clavel, J., Julliard, R., Lavergne, S., Mouillot, D., Julliard, R., Thuiller, W., Venail, P., Villeger, S., \& Mouquet, N. 2010. Defining and measuring ecological specialization. Journal of Applied Ecology 47:15-25.

Figueroa-Rojas, R.A., Corales, E.S. \& Alvarado, S. 2003. Diet of the Red-Backed Hawk (Buteo polyosoma) in a forested area of the Chilean Patagonia and its relation to the abundance of rodent prey. El Hornero 18(1):43-52.

Fuentes, M.A., Simonetti, J.A., Sepúlveda, M.A. \& Acevedo, P.A. 1993. Diet of the Red-backed Buzzard (Buteo polyosoma exsul) and the Short-eared Owl (Asio flammeus suinda) in the Juan Fernández archipielago of Chile. Journal of Raptor Research 27:167-169.

Futuyma, D.J. \& Moreno, G. 1988. The evolution of ecological specialization. Annual Review of Ecology and Systematic 19:207-233.

Gajardo, R. 1994. La vegetación natural de Chile: Clasificación y distribución geográfica. Editorial Universitaria (Chile). $165 \mathrm{pp}$.

Goodwall, J.D., Johnson, A.W. \& Phillipi, R.A. 1993. Las aves de Chile, su conocimiento y sus costumbres. Volumen II, Platt
Establecimientos Gráficos, Buenos Aires. pp.

Hutchinson, G.E. 1957. Concluding remarks. Cold Spring Harbor Symposium. Quantitative Biology 22:415-427.

JiMÉNEZ, J. 1995. Historia natural del aguilucho Buteo polyosoma: una revisión. El Hornero 14:1-9.

Juliá, C., Montesinos, S. \& Maldonado, A. 2008. Características climáticas de la Región de Atacama En: Libro Rojo de la Flora Nativa y de Los Sitios Prioritarios para su Conservación: Región de Atacama (Eds. Squeo, F.A., Arancio, G. \& Gutiérrez, J.R.), pp. 3: 25-42. Ediciones Universidad de La Serena, La Serena, Chile.

KASSEN, R. 2002. The experimental evolution of specialists, generalists, and the maintenance of diversity. Journal of Evolutionary Biology 15:173-190.

Levins, R. 1968. Evolution in Changing Environments. Princeton University Press, Princeton, NJ. 123 pp.

Monserrat, A.L., Funes, M.C. \& Novaro, A.J. 2005. Respuesta dietaria de tres rapaces frente a una presa introducida en Patagonia. Revista Chilena de Historia Natural 78:425-439.

Muñoz-Pedreros, A. \& GIL, C. 2009. Orden Rodentia. En: Mamíferos de Chile (Eds. Muñoz-Pedreros \& Yáñez), pp. 93-157. CEA Ediciones.

Muñoz-Pedreros, A., Rau, J. \& Yáñez, J. 2004. Estudio de Egagrópilas en aves rapaces. Pp. 265-279, En: Muñoz A., Rau J. \& Yañez J. (eds). Aves Rapaces de Chile. CEA Ediciones, Valdivia, Chile.

Novoa, J.E., Tracol, Y. \& Lopez, D. 2008. Paisajes EcoGeográficos de la Región de Atacama 2: 13-24. En: Libro Rojo de la Flora Nativa y de Los Sitios Prioritarios para su Conservación: Región de Atacama. Squeo, F.A., Arancio, G. \& Gutiérrez, J.R. (Eds.) Ediciones Universidad de La Serena, La Serena, Chile.

Olrog, C.C. 1959. Las Aves argentinas, una guía de campo. Universidad Nacional de Tucumán, Instituto Miguel Lillo, Tucumán, Argentina.

PAcheco, J.F. 2004. Ocorrência acidental de Buteo polyosoma (Quoy \& Gaimard, 1824) na Ilha de Cabo Frio, Rio de Janeiro, Brasil. Ararajuba 12(2):168-169.

Pavez, E.F. 2004. Descripción de las Aves Rapaces Chilenas. Pp. 29-103, En: Muñoz, A., Rau, J. \& Yañez, J. (eds). Aves Rapaces de Chile. CEA Ediciones, Valdivia, Chile.

Raimilla, V., RAU, J.R. \& MuÑoz- Pedreros, A. 2012.Estado del arte del conocimiento de las aves rapaces de Chile: Situación actual y proyecciones futuras. Revista Chilena de Historia Natural 85: 469-480.

Schlatter, R.P., YÁÑEz, J.L. \& JaKsic, F.M. 1980. Food niche relations between Chilean Eagles and Red Backed Buzzards in Central Chile. Auk 97: 897-898.

Spotorno, A.E., Zuleta, C., Gantz, A., Saiz, F., Rau, J., Rosenmann, M., Cortés, A., Ruiz, G., Yates, L., Couve, E. \& Marin, J.C. 1998. Sistemática y Adaptación de Mamíferos, aves e insectos fitófagos de la Región de Antofagasta, Chile. Revista Chilena de Historia Natural 71: 501-526.

Spotorno, A.E., Zuleta, C., Walker, G., Manriquez, S., VAlladares, P. \& Marín, J.C. 2013. A small, new gerbilmouse Eligmodontia (Rodentia: Cricetidae) from dunes at the coasts and deserts of north-central Chile: molecular, chromosomic, and morphological analyses. Zootaxa 3683(4): 377-394.

Squeo, F.A., Arancio, G., Gutiérrez, J.R., Letelier, L., Arroyo, 
Diet of Variable Hawk from Atacama Desert, Chile: Pablo Valladares Faúndez ET AL.

M.T.K., León- Lobos, P. \& Rentería- Arrieta, L. 2008. Sitios Prioritarios para la Conservación de la Flora Nativa Amenazada de la Región de Atacama 2: 13-24. En: Libro Rojo de la Flora Nativa y de Los Sitios Prioritarios para su Conservación: Región de Atacama. Squeo, F.A., Arancio, G. \& Gutiérrez, J.R. (Eds.) Ediciones Universidad de La Serena, La Serena, Chile.

Thiollay, J.M. 1994. Family Accipitridae (Hawks and Eagles). In: del Hoyo, J.M., Elliot, A., Sargatal, J. (Eds.), Handbook of the Birds of the World: New World Vultures to Guineafowl. Lynx Edicions, Barcelona, pp. 52-205.

Travaini, A., Santillán, M.A. \& Zapata, S.C. 2012. Diet of the Red-backed Hawk (Buteo polyosoma) in two environmentally contrasting areas of Patagonia. Studies on Neotropical Fauna and Environment 47(1): 1-8
Valladares, P. 2011. Análisis, síntesis y evaluación de la literatura de lagartos de la Región de Atacama, Chile. Gayana 75(1): $81-98$

Valladares, P. 2012. Mamíferos terrestres de la Región de Atacama, Chile. Comentarios sobre su distribución y estado de conservación. Gayana 76(1): 13-28.

Valladares, P. \& C. CAmpos. 2012. New record of Abrocoma bennetti murrayi (Rodentia, Abrocomidae) from the Atacama region. Extension of distribution range in Chile. IDESIA 30(2): 115-118.

VAURIE, C. 1962. A systematic study of the Red-backed Hawks of South America. Condor 64: 277-290.

Yáñes, J., Tamayo, M., NúÑez, H. \& Sanino G.P. 2009. Clave de determinación, Pp. 423. En: Mamíferos de Chile, MuñozPedreros \& Yáñez (Eds.).

Recibido: 02.06.14

Aceptado: 17.08 .15 